\title{
Configurações
}

Revista de sociologia

\section{A intervenção técnica junto de crianças em acolhimento residencial em casa de abrigo para vítimas de violência doméstica}

Technical intervention with children in residential shelter for victims of domestic violence

Intervention technique auprès des enfants dans les refuges pour les victimes de violence domestique

\section{Ana Sani e Ana Lúcia Correia}

\section{CpenEdition}

\section{Journals}

Edição electrónica

URL: http://journals.openedition.org/configuracoes/7214

DOI: 10.4000/configuracoes. 7214

ISSN: 2182-7419

Editora

Centro de Investigação em Ciências Sociais

Edição impressa

Paginação: 138-158

ISSN: 1646-5075

\section{Refêrencia eletrónica}

Ana Sani e Ana Lúcia Correia, «A intervenção técnica junto de crianças em acolhimento residencial

em casa de abrigo para vítimas de violência doméstica », Configurações [Online], 23 | 2019, posto online no dia 28 junho 2019, consultado o 30 junho 2019. URL : http://journals.openedition.org/ configuracoes/7214; DOI : 10.4000/configuracoes.7214 
Sani, Ana; Correia, Ana Lúcia - A intervenção técnica junto de crianças em acolhimento residencial em casa de abrigo para vítimas de violência doméstica. Configurações, vol. 23, 2019, pp. 138-158.

\title{
A intervenção técnica junto de crianças em acolhimento residencial em casa de abrigo para vítimas de violência doméstica
}

ANA SANI *

Faculdade de Ciências Humanas e Sociais da Universidade Fernando Pessoa, Porto

ANA LÚCIA CORREIA**

Universidade Fernando Pessoa. Agrupamento de Escolas de Almeida, Guarda

\section{Resumo}

Apresenta-se um estudo qualitativo que analisou, na perspetiva dos técnicos, a intervenção preconizada junto de crianças residentes temporariamente em casa de abrigo para vítimas de violência doméstica. A amostra intencional foi composta por 11 técnicos de nove casas de abrigo em Portugal, os quais responderam a uma entrevista estruturada, cujo guião com questões foi disponibilizado eletronicamente. Os resultados revelaram a primazia da intervenção junto das mães, orientada para o treino de competências parentais, vinculação, práticas educativas, regras e rotinas a usar com os seus filhos. A intervenção direta com as crianças é secundarizada por falta de técnicos, as especificidades do contexto e a formação especializada exigida. Sublinha-se a importância de uma ação focada também na criança, visando a promoção do seu bem-estar e desenvolvimento ajustados.

Palavras-chave: Casas de abrigo, crianças, violência, intervenção.

\begin{abstract} violence

\footnotetext{
*E-mail: anasani@ufp.edu.pt

**E-mail: ana.arandapsi@gmail.com
}

Technical intervention with children in residential shelter for victims of domestic

A qualitative study accounting the technician's perspective and analysing the intervention recommended to children that are temporarily in domestic violence shelters is presented. The intentional sample consisted of 11 technicians from nine domestic violence shelters in Portugal, who answered a structured interview, whose script with questions was made available electronically. The obtained results indicate the priority 
of intervention among mothers, oriented to the training of parental skills, attachment, educational practices, rules and routines to use with their children. Direct intervention with children is secondary because of the lack of technicians, the specificities of the context and the specialized training required. The importance of action focused on the child is stressed, aiming to promote adjusted well-being and development.

Keywords: Shelters, children, violence, intervention.

\section{Résumé \\ Intervention technique auprès des enfants dans les refuges pour les victimes de vio- lence domestique}

Nous présentons une étude qualitative qui visait analyser, du point de vue des techniciens, l'intervention recommandée auprès des enfants résidant temporairement dans des refuges pour victimes de violence domestique. L'échantillon intentionnel était constitué par onze techniciens de neuf refuges au Portugal, qui ont répondu à une interview structurée, dont le script avec des questions a été mis à disposition sous la forme électronique. Les résultats ont révélé la primauté de l'intervention chez les mères, orientée vers la formation des compétences parentales, l'attachement, les pratiques éducatives, les règles et les routines, à utiliser avec leurs enfants. L'intervention directe auprès des enfants est secondaire en raison du manque de techniciens, des spécificités du contexte et de la formation spécialisée requise. L'importance de l'action centrée sur l'enfant est également soulignée, visant à promouvoir leur bien-être et leur développement.

Mots-clés: Refuges, enfants, violence, intervention.

\section{Introdução}

As casas de abrigo para vítimas de violência doméstica são por definição (Lei n.o $107 / 99$ regulamentado pelo Decreto-Lei no 323/2000) espaços de segurança, criados para receber e apoiar em termos residenciais, ainda que de um modo transitório, adultos e crianças em situação de especial vulnerabilidade e risco de revitimação. Não obstante estes equipamentos de resposta social assentarem neste objetivo comum e partilharem muitos outros aspetos relativos ao seu funcionamento (cf. Decreto Regulamentar $\mathrm{n}^{\circ} 1 / 2006$, alterado pelo Decreto Regulamentar n. ${ }^{\circ} 2 / 2018$; Lei n. ${ }^{\circ} 112 / 2009$, alterada pela Lei n. ${ }^{\circ}$ 129/2015), existem algumas especificidades (Correia e Sani, 2015; Magalhães, Morais, \& Castro, 2011). Uma das especificidades prende-se com a possibilidade de acolhimento de crianças, constituindo-se assim as casas de abrigo, uma entre outras das estruturas possíveis de acolhimento residencial, ainda que não pertencentes ao sistema de promoção e proteção (cf. Instituto de Segurança Social, 2017). Neste sentido, muito embora o acolhimento em casa de abrigo represente uma resposta interventiva especializada às necessidades, interesses e direitos de crianças e jovens, e devam estas estruturas promover a sua 
educação, bem-estar e desenvolvimento integral (Martins, 2016), a integração repentina num ambiente alternativo é um desafio cheio de adaptações para a criança. Mesmo que seja assegurado um dos mais básicos princípios orientadores e fins de intervenção (cf. Delgado e Gersão, 2018) que deve caracterizar a resposta social fornecida pelas estruturas do sistema de promoção e proteção (e.g., a continuidade de relações de afeto e qualidade significativas, no caso as mães), estar acolhida em casa de abrigo está longe de ser uma alternativa adequada, ainda que de "curta duração", para o bem-estar e desenvolvimento da criança em qualquer faixa etária. Estarão as casas de abrigo preparadas para receber as crianças expostas à violência doméstica, proporcionando uma intervenção que responda adequadamente às necessidades, aos interesses e aos direitos de crianças e jovens? Assim, é sobre a população de crianças e jovens acolhidas em casa de abrigo e a respeito da intervenção operada junto destas que centraremos a atenção neste artigo.

\section{As crianças em acolhimento temporário em casa de abrigo}

A literatura vem estimando que as crianças superam, em número, o de vítimas adultas acolhidas por violência doméstica (Chanmugam, 2016; Hogan e O'Reilly, 2007; Williamson, 2006), na medida em que muitas das mães que integram as casas de abrigo trazem consigo os seus filhos (Cunningham e Baker, 2004; Øverlien, 2011). Atendendo a esta evidência deverão os serviços e os programas prestados às vítimas adultas de violência doméstica em casas de abrigo estenderem-se também aos filhos, sob pena do apoio prestado não dar resposta a uma das maiores necessidades experienciadas pelas vítimas, o apoio ao bem-estar das suas crianças.

Estudos internacionais (e.g., Saathoff e Stoffel, 1999; Williamson, 2006) revelam como em certos países, há já algumas décadas, várias casas de abrigo promovem programas destinados a colmatar as necessidades de saúde mental, de saúde em geral, de educação e de segurança das crianças residentes. Saathoff e Stoffel (1999) referem inclusive, a propósito de um estudo realizado em 1997 pela National Coalition Against Domestic Violence, que $72 \%$ destes equipamentos de resposta social às vítimas de violência doméstica ofereciam, nessa altura, aquele tipo de serviços dirigidos também às crianças. As crianças e adolescentes expostos à violência doméstica entre pais estão numa situação de elevado risco, não apenas de vitimação direta (e.g., Bourassa, Lavergne, Damant, Lessard e Turcotte, 2006; Jouriles, McDonald, Slep, Heyman e Garrido, 2008), mas de serem afetados negativamente no seu desenvolvimento. De acordo com diversos estudos (Evans, Davies e Dilillo, 2008; Galiano e Duarte, 2011; Jouriles, Rosenfield, MacDonald, Mueller, 2014; Muñoz, Bello, Sandoval, Romer e Nieto,2016) o impacto negativo desta experiência pode manifestar-se em sinais e sintomas mais ou menos visíveis, quer de internalização (e.g., 
tristeza, culpa, preocupação, queixas somáticas, ansiedade, depressão), quer de externalização (e.g., impulsividade, agressividade). As consequências da exposição à violência doméstica podem manifestar-se de forma diferenciada nas crianças, atendendo a variáveis mediadoras (e.g., individuais, familiares, contextuais, situacionais) (McDonald e Grych, 2006; Evans, Davies e Dilillo, 2008; Holt, Buckley e Whelan, 2008; Soares e Sani, 2015). A persistência desses sintomas pode dar origem ao diagnóstico de perturbações traumáticas (Margolin e Vickman, 2007) ou ser altamente desorganizadora do comportamento geral da criança, predispondo-a mais para a agressão física e conduta desviante (Galiano e Duarte, 2011), para uma maior tendência de envolvimento futuro em relacionamentos violentos, quer como um perpetrador, quer como uma vítima (Moretti, Bartolo, Craig, Slaney, Odgers, 2014; Mandal e Hindi, 2015).

O apoio prestado às crianças é muito necessário, não apenas pelo impacto negativo da exposição à violência doméstica (Sani, 2004), mas também pela adaptação a um novo contexto residencial.

\section{Adaptação ao contexto de um acolhimento residencial temporário}

Para as crianças, mesmo tratando-se de um acolhimento temporário, há todo um contexto de novas adaptações (Bowyer, Swanston e Vetere, 2015), quer às pessoas que lá se encontram, quer ao ambiente em si, com novas regras, por norma, bem diferentes das do contexto donde provêm. Esta adaptação a um novo contexto pode, em si, constituir também um novo e positivo desafio, como pode ser percebido como uma nova ameaça (Chanmugam, 2016, 2017). Em alguns casos, esse risco resulta das dificuldades em garantir, neste período de transição, a segurança da vítima e das crianças, quando, agora libertos da ameaça do agressor, tudo devem fazer para não por em causa essa segurança, incluindo não contactar com familiares ou amigos. E, portanto, este desafio de se verem privados, ainda que temporariamente e por razões compreensíveis, de possíveis figuras de suporte, gera por vezes sentimentos que conduzem a atuações que podem ameaçar a sua segurança e a dos seus.

De igual modo, em contexto de casa de abrigo, o ambiente educativo em que se estabelecem as relações entre progenitor e criança passa a ser diferente. Para as vítimas adultas exercer a parentalidade neste novo meio é uma tarefa desafiante, na medida em que provêm de um contexto de controlo e poder, onde frequentemente se viram desautorizadas e com grandes dificuldades em manter uma aliança segura com as suas crianças (Sani, 2008). Para os filhos, esta nova situação pode ser uma oportunidade de restabelecimento ou fortalecimento de laços com o progenitor que o acompanha ou, pelo contrário, um 
campo aberto para esgrimir as suas angústias e revolta pela situação em que se encontram.

As crianças residentes em casas de abrigos são assim uma população muito especial, que além da vivência da violência doméstica, se confrontam agora com a realidade da adaptação a um contexto provisório de acolhimento. Por essa razão importa considerar que, além do stresse experienciado pela exposição à violência doméstica dos seus cuidadores, estas crianças tendem a expressar outras manifestações de impacto por estas adaptações transitórias que têm de enfrentar. É por isso, de especial relevância, que o acolhimento de crianças, provindas de situações de violência na família, prime pela qualidade, que lhes possibilite bem-estar, segurança e, sempre que possível, a otimização de competências, aspetos que podem concorrer para o desenvolvimento integral e para um futuro promissor (Sani e Caridade, 2016).

Por vezes o perigo é iminente e a resposta a dar para as situações de elevado risco não se coaduna com tempos de preparação para a mudança. A entrada para uma casa de abrigo é, na maioria das vezes, constrangida por vários fatores, não apenas pelo tempo e pela urgência de se tomarem decisões acertadas, que protejam e salvaguardem os direitos e interesses de todos, mas por condições associadas à estrutura e aos recursos técnico-profissionais apropriados para avaliar, programar e implementar intervenções neste contexto (Amaral e Martins, 2014). Parece assim evidente que devem ser pensadas, quer antes, quer por altura do acolhimento, as melhores estratégias para que esta entrada e permanência temporária numa casa de abrigo não sejam vividas como momentos de crise difíceis, designadamente por crianças e jovens vítimas de violência (cf. Sani e Caridade, 2016). A intervenção com esta população deve contribuir para potenciar competências, prevenir o impacto negativo da violência doméstica e ajudar a suprimir a probabilidade de contacto da criança com o sistema de proteção e, em última instância, a entrada dos jovens no sistema de justiça (Schechter e Knitzer, 2004).

Nas últimas décadas, a emergência de um conhecimento fundamentado na experiência de inúmeros serviços e na investigação empírica junto das vítimas de violência, tem permitido que hoje se defenda afincadamente o quão importante pode ser a intervenção, conscienciosa dos múltiplos fatores de risco exibidos por estas famílias, para o futuro de cada uma e para a sociedade. Para as crianças, a intervenção pode significar a diferença entre ter ou não garantidos os seus direitos à saúde, à segurança, à educação, ao bem-estar integral, a uma vida sem violência, usufruindo para tal, e se necessário, de cuidados especializados. Graham-Bermann (2001) numa revisão realizada a 15 projetos centrados na intervenção junto de crianças expostas à violência doméstica concluiu que estas intervenções são de especial relevância para a convivência e bem-estar dos envolvidos. 
Desta forma as crianças acolhidas em casa de abrigo podem e devem beneficiar de propostas dirigidas diretamente a elas ou aos adultos que as acompanham neste acolhimento (Chanmugam, 2017). A entrada na casa de abrigo deve ser também preparada ou pelo menos apoiada, podendo ser criados materiais ou realizadas atividades que a favoreçam. Para o acolhimento decorrer de forma segura devem as crianças conhecer e participar na elaboração de planos de segurança. É necessário que as crianças sejam capazes de identificar as pessoas em quem podem, realmente, confiar no seu círculo social, bem como desenvolver um plano de segurança quando sentem que podem experimentar ou testemunhar violência (Chanmugam e Hall, 2012; Poole, Beran e Thurston, 2008). Outras das propostas em contexto de acolhimento pode passar pela criação de espaços psicoeducativos que permitam às crianças e aos jovens abordar, individualmente ou em grupo, as suas experiências e encontrar novos referenciais (Campanón, 2008). A instituição deve centrar a sua atenção nos processos relativos à personalização e promover atividades orientadas para bom acolhimento, a escuta, a observação sistemática, a elaboração de um projeto educativo individualizado, dando às crianças a oportunidade de alcançar os sistemas de participação e de socialização, a educação, a responsabilidade, a autonomia, a liberdade, ao valor do quotidiano no processo de crescimento (Del Valle \& Zurita, 2005).

Paralelamente as crianças podem integrar grupos envolvidos em sessões de aconselhamento ou realizar intervenções diádicas (e.g., com a mãe; técnicos) que contribuam para a diminuição de problemas evidenciados (e.g., agressão, ansiedade, depressão) e favoreçam as relações interpessoais com outras crianças. Essas atividades podem ainda permitir o expressar de emoções, o modelar de atitudes e crenças, o ensaiar de comportamentos não violentos, cometer erros e aprender através deles (Rivett, Howarth e Harold, 2006). Para tal podem ser usadas estratégias e técnicas como a arte, a escrita, a representação, o jogo, o vídeo, as conversas com um adulto, as atividades de grupo estruturadas, o teatro de fantoches, a música, os exercícios de movimento, o conto de histórias e a expressão verbal, entre outras (Thompson e Trice-Black, 2012).

Alguns programas adaptados de propostas internacionais foram elaborados e testados, tendo em termos de estrutura sido replicados com resultados satisfatórios, no âmbito de serviços e projetos dirigidos para a intervenção com crianças expostas à violência doméstica (cf. Sani, 2018). Desde há décadas que a literatura (e.g., Graham-Bermann \& Hughes, 2003; Wagar \&. Rodway, $1995)$ tem evidenciado como as propostas de prevenção terciária, dirigidas aos filhos de vítimas de violência doméstica, se mostram eficazes para responder aos problemas comportamentais (e.g., agressividade, ansiedade), assim como contribuem para reforçar naquelas o conhecimento e o uso de estratégias de confronto para lidar com a violência. A intervenção em grupo, em particular, tem mostrado inúmeras potencialidades ao permitir que a criança quebre o 
segredo e descubra outros casos como o seu (Jaffe, Wolfe, \& Wilson, 1990; Peled \& Davis, 1995), além de possibilitar trabalhar conjuntamente com outras crianças vítimas aspetos relativos à violência, à segurança, à resolução do conflito (Ericksen \& Henderson, 1998).

Em vários outros projetos (e.g., Domestic Violence Shelter Screening Project; Honor Our Voices project) podemos encontrar sugestões para intervir de forma satisfatória para suprimir as necessidades de crianças e jovens acolhidos em casa de abrigo (Ahsan, 2004; Center for Child and Family Health, 2010; Edleson, Nguyen e Kimball, 2011; Schechter e Knitzer, 2004). De modo geral, as propostas passam por promover a formação do pessoal da casa de abrigo, sobretudo os que interagem mais frequentemente com a criança, por desenvolver programas de apoio à parentalidade positiva, ou por criar uma rede de suporte comunitário com profissionais de áreas multidisciplinares que potenciem a resolução de questões específicas, garantam segurança e estabilidade necessárias (Sani, no prelo).

Operar transformações nestes contextos de acolhimento temporário como são as casas de abrigo para vítimas de violência doméstica pressupõe compreender quais as necessidades manifestadas por utentes, sejam estes adultos (Faro e Sani, 2014) ou crianças, assim como conhecer a perceção dos técnicos que atuam neste contexto específico, que procura responder socialmente ao problema da violência nas famílias.

Assim, apresenta-se de seguida um estudo que teve como questão de partida: quais as práticas interventivas utilizadas pelos técnicos de casas de abrigo, a nível nacional, junto de crianças filhas de mães vítimas de violência doméstica? Este trabalho inseriu-se numa investigação alargada que entre outros pretendeu atingir os seguintes objetivos: i) caracterizar as casas de abrigo em Portugal, e ii) descrever a intervenção técnica exercida em casas de abrigo, designadamente com as crianças. No presente artigo serão abordados os resultados obtidos relativamente ao segundo objetivo.

\section{Estudo sobre as práticas interventivas junto de crianças em casas de abrigo}

\subsection{Método}

Para a concretização do estudo constituiu-se uma amostra intencional com 11 técnicos, de ambos os sexos e com idades compreendidas entre os 28 e os 49 anos de idade, os quais exerciam funções em nove casas de abrigo no território português (cf. Quadro 1). Para a inclusão dos participantes estabeleceram-se dois critérios fundamentais: i) tratar-se de casas de abrigo nacionais que alojassem, simultaneamente, mulheres e crianças vítimas de violência doméstica; ii) os técnicos a responder ao inquérito tinham de exercer funções em casas de 
abrigo há pelo menos seis meses. Os participantes eram na maioria do sexo feminino $(n=10)$, com formação nas áreas da psicologia $(n=6)$ e com tempo médio de funções de seis anos

Quadro 1.

Dados sociodemogr áficos relativas aos técricos de casa de abrigo

\begin{tabular}{lllllll}
\hline Técnicos & $\begin{array}{l}\text { Casa de } \\
\text { abrigo }\end{array}$ & Sexo & Idade & Habilitaçōes & Função & $\begin{array}{l}\text { Tempo de } \\
\text { função }\end{array}$ \\
\hline S1 & C1 & F & 39 & Lic. Serviço Social & Diretora Técrica & 6 anos \\
S2 & C1 & M & 33 & Msc. Medicina Legal & Psicólogo & 5 anos \\
S3 & C2 & F & 32 & Lic. Psicologia & Psicologa & 9 meses \\
S4 & C3 & F & 28 & Msc. Psicologia & Diretora Técrica & 13 meses \\
S5 & C4 & F & 36 & Lic. Psicologia & Dirctora Tecrica & 8 anos \\
S6 & C5 & F & 36 & Lic. Psicologia & Diretora Técrica & 9 anos \\
S7 & C6 & F & - & Lic. Psicologia & Psicóloga & 7 anos \\
S8 & C7 & F & 37 & Lic. Sociologia & Socióloga & 14 anos \\
S9 & C8 & F & 30 & Lic. Psicologia & Coord.Psicóloga & 5 anos \\
S10 & C6 & F & 49 & Lic. Serviço Social & Assistente Social & 7 anos \\
S11 & C9 & F & 47 & Lic. Educação Social & Educadora Social & 10 anos \\
\hline
\end{tabular}

Legenda: Lic. (licenciado); Msc(mestre); Coord. (coordenedore)

Em termos procedimentais, os dados foram recolhidos através do método de inquérito por entrevista estruturada em formulário próprio, disponibilizada eletronicamente. Para tal foi construído um guião com questões abertas e fechadas respeitantes a dados sociodemográficos (e.g., Idade, Sexo, Estado civil, Habilitações), às características da casa de abrigo (e.g., Caracterize brevemente a instituição em que trabalha; Quais as características da casa de abrigo - dimensão, estruturas, equipamentos, capacidade?) e à atividade operativa dos técnicos (e.g., Quais as funções que desenvolve na casa de abrigo; Como descreve a intervenção desenvolvida junto dos utentes da casa de abrigo?).

Previamente, procedeu-se ao contacto telefónico com as casas de abrigo, com o objetivo de dar conhecimento do estudo e de pedir informações, junto do diretor técnico, acerca da forma como deveríamos proceder relativamente ao pedido de autorização. O e-mail enviado para a direção técnica de cada casa de abrigo continha toda a explicação acerca da natureza do estudo, da população a quem eram dirigidas as entrevistas, a menção de garantia de anonimato e confidencialidade dos dados obtidos, seguido de um pedido de consentimento informado e indicação sobre o modo como deveriam proceder para reencaminhar as respostas e para quem. Após a obtenção do consentimento informado para o estudo, cada técnico poderia avançar para o preenchimento da entrevista em formato digital. A CIG - Comissão para a Cidadania e a Igualdade de Género - apoiou a divulgação do estudo às casas de abrigo a nível nacional, de modo a reforçar a sua pertinência. A recolha dos dados decorreu entre os 
meses de abril e outubro de 2012, tendo o processo de codificação sido concluído em julho de 2013.

Os dados obtidos foram sujeitos à análise de conteúdo (Bardin, 2006), tendo sido construídas, atendendo aos objetivos definidos, algumas categorias prévias (categorização por caixas), que possibilitassem agrupar e classificar as narrativas produzidas pelos entrevistados de acordo com os temas questionados. Para tal recorremos ao uso da frase como unidade de análise. Posteriormente, realizámos uma classificação analógica e progressiva do material empírico, utilizando-se uma codificação aberta das entrevistas, fazendo com que a designação das subcategorias emergisse dos dados e seguisse de perto o registo semântico presente nas narrativas dos participantes (Strauss e Corbin, 1998). As categorias foram posteriormente objeto de validação por um segundo codificador, que procedeu à análise do mesmo material, tendo por referência a categorização, previamente discutida. De seguida serão apresentadas as categorias emergentes (cf. Quadro 2), expondo-as de forma integrada com a identificação dos respondentes e explicitação apenas de alguns excertos dos relatos de técnicos, para validar o discurso dos participantes e evitar redundância e repetições de conteúdo. Dada a extensão do material obtido e a impossibilidade de expor todas as narrativas, foram usados para ilustrar os excertos que nos pareceram clarificadores.

\section{Resultados}

Quadro 2. Categorias, subcategorias e principais resultados

\begin{tabular}{|c|c|c|}
\hline Categoria & Subcategorias & Principais resultados \\
\hline \multirow{6}{*}{$\begin{array}{l}\text { Práticas } \\
\text { Interventivas } \\
\text { dos Técnicos }\end{array}$} & $\begin{array}{l}\text { Intervençăo com os } \\
\text { utentes }\end{array}$ & $\begin{array}{l}\text { Mais às mutheres: Intervençäo } \\
\text { multidisciplinar e temporária, } \\
\text { durecionada para as suas necessidades } \\
\text { (e.g. descnvolvimento de competéncias } \\
\text { de autonomizaçāo, da autocstima e do } \\
\text { autoconceito; rolaçāo com os filhos } \\
\text { - Menos às crianças: Educaçào e saúde; } \\
\text { Trabathar a autoestima e autoconceito }\end{array}$ \\
\hline & $\begin{array}{l}\text { Necessidades das } \\
\text { criancas acolhidas }\end{array}$ & $\begin{array}{l}\text { - Proteção, segurança, } \\
\text { - Acompanharnento escolar e psicológico, } \\
\text { - Atençāo, afeto } \\
\text { - Imposiçăo de regras e limites } \\
\text { - Cuicados de saùde }\end{array}$ \\
\hline & $\begin{array}{l}\text { Competéncias para a } \\
\text { intervencâo com as } \\
\text { criancas }\end{array}$ & $\begin{array}{l}\text { - Conhecimentos teoricos } \\
\text { - Competências técuicas } \\
\text { - Compctēncias relacionais }\end{array}$ \\
\hline & $\begin{array}{l}\text { Intervençio } \\
\text { desenvolvida com as } \\
\text { crianças na casa de } \\
\text { abrigo }\end{array}$ & $\begin{array}{l}\text { - Acompanhamento } \\
\text { - Encaminhamentos } \\
\text { - Integraçào } \\
\text { - Atividades lúdicas }\end{array}$ \\
\hline & $\begin{array}{l}\text { Dificuldades/desafios } \\
\text { na intervençāo com } \\
\text { crianças }\end{array}$ & $\begin{array}{l}\text { - Dificuldades: Experiēncia de } \\
\text { acolhimento; Burocracias institucionais; } \\
\text { Consequencias da exposição; Orgâos } \\
\text { judiciais; Comportamentos đesajustados } \\
\text { - Desafios: Implementaça de uma } \\
\text { intervenção em grupo }\end{array}$ \\
\hline & $\begin{array}{l}\text { Sugestōes na } \\
\text { intervençāo com } \\
\text { criangas }\end{array}$ & $\begin{array}{l}\text { - Formaçāo profissional } \\
\text { - Reforço de profis sionais } \\
\text { - Intervenção com as crianças } \\
\text { - Apoio de outras entidades }\end{array}$ \\
\hline
\end{tabular}




\subsection{Intervenção com os utentes}

O apoio dos técnicos que acompanham estas mulheres foi descrito como muito importante nesta fase de adaptação pela qual elas estão a passar. Segundo os técnicos (S2, S3, S7, S9), a intervenção realizada junto dos utentes estava dependente dos recursos existentes no momento. Assim a intervenção era feita considerando "a equipa técnica, que é uma equipa multidisciplinar" (S2) e tendo em conta que era "uma intervenção temporária” (S1) pois o período de permanência nas casas de abrigo era de 6 meses, estipulado por lei (D.R. 1/2007 de 25 de janeiro), não havendo acompanhamento após a saída da utente da casa de abrigo.

Durante o tempo de permanência, a intervenção realizada pelos técnicos (S5, S6, S7, S8, S9, S11) era feita caso a caso _ "necessidades específicas de cada agregado, sendo comum a todas a capacitação das mulheres para a autonomização e responsabilização enquanto cidadãs ativas na comunidade.” (S6). A intervenção ainda que temporária era definida por objetivos gerais comuns às várias casas: "potenciar e desenvolver competências; desencadear mecanismos que lhes permitam seguir um novo projeto de vida, sem violência, de forma sustentada e numa perspetiva de autonomização" (S1).

Segundo os técnicos (S2, S3, S4, S6, S8) a intervenção era dirigida diretamente e, sobretudo, às mulheres - "na sua relação mãe - filhos, o que tem um impacto muito positivo na mudança comportamental das crianças” (S5). O objetivo era que essas mães se tornassem capazes de lidar com os seus filhos, ganhar autoridade, apoiá-los a nível escolar, social, afetivo e no relacionamento com os pares. Como referiu um dos técnicos:

\footnotetext{
Tentamos muitas das vezes trabalhar competências parentais cá dentro, tentar sensibilizaras mães em termos afetivos, em termos de práticas educativas, o acompanhamento escolar, o facto de cumprir com as refeições, cumprir com os planos de saúde, cumprir com as regras, com as rotinas em relação aos filhos, tudo isso é feito, é feito um trabalho em parceria comas mães e que nos cabe a nós também, fazer essa monitorização. (S3)
}

Os técnicos procuraram ainda, em conjunto com as mães, que a casa de abrigo fosse um ponto de viragem, fazendo uso de uma abordagem que fosse "promotora de competências pessoais, profissionais, parentais e sociais" (S11). Assim, os técnicos procuraram assegurar uma intervenção mais individualizada, por exemplo, de "promoção da autoestima das mulheres e crianças e desenvolvimento de um autoconceito positivo" (S5) sendo igualmente trabalhadas pela equipa técnica de modo a que esta família pudesse superar esta fase e traçar um novo projeto de vida. Neste sentido, várias áreas foram definidas 
para a intervenção, tais como "educação, saúde, profissional, jurídica e psicossocial (mulher, criança e família)" (S10).

\subsection{Necessidades das crianças acolhidas}

Segundo os técnicos (S1, S3, S4, S7, S10) as necessidades evidenciadas pelas crianças aquando da sua entrada na casa de abrigo prendiam-se com questões relacionadas com a proteção, segurança e acompanhamento escolar e psicológico - "dependendo de diferentes variáveis, nomeadamente idade, impacto, desenvolvimento, resiliência e outras, assinalaria que a necessidade mais evidenciada é a segurança” (S10). Como foi referido por alguns técnicos (S6, S7, S9), estas crianças precisavam sentir que não estão sozinhas ("Em muitas situações observa-se a necessidade de atenção, afeto" - S6), necessitavam da atenção das mães, de continuar a relacionar-se com os familiares, incluindo com o pai, que na maioria dos casos era o agressor e também com o grupo de pares.

As alterações de comportamento foram também referenciadas pelos técnicos, que achavam que devido aos problemas que enfrentaram, estas crianças sentiam-se debilitadas, revoltadas e há que estar atento ao seu comportamento, daí a importância das suas mães imporem limites e regras ("Em relação às crianças as necessidades evidentes são a falta de rotinas, regras”, S9).

De acordo com os técnicos, as crianças que chegam à casa de abrigo exprimiam uma panóplia de sentimentos (e.g., culpa, revolta, baixa autoestima), daí que alguns técnicos $(S 3, S 4, S 9)$ referiram também a necessidade de cuidados de saúde - "As principais necessidades, de forma geral, destas crianças estão relacionadas com a vinculação afetiva, com o sentimento de culpa (...). É possível verificar em algumas crianças, a necessidade de trabalhar as emoções” (S2).

\subsection{Competência para a intervenção com as crianças.}

Todos os participantes consideraram ser necessário conhecimentos teóricos, competências técnicas e relacionais para se poder intervir junto de crianças direta e/ou indiretamente vítimas de violência doméstica. Em termos concetuais, os técnicos referiram ser primordial ter conhecimento acerca da temática da violência doméstica, do estádio de desenvolvimento de uma criança, do funcionamento familiar, assim como possuir competências na área social e humana, no que diz respeito ao trabalho com crianças vítimas de violência interparental acolhidas em casa de abrigo. Na maioria das respostas (S2, S3, S4, S6, S7, S8, S9, S10, S11) a relação empática era apontada como a competência mais importante no trabalho com estas crianças.

É extremamente importante ter a capacidade de conseguirmos sentir o que elas sentem, colocarmo-nos no lugar delas - criarmos empatia - para conseguirmos trabalhar os aspetos psicoemocionais de 
relevo. Ter conhecimento da evolução psicoemocional da criança, para poder fazer a distinção do normal e patológico. E, como é claro, ter conbecimento da temática - Violência Doméstica - para adequarmos os conbecimentos científicos para uma melhor intervenção (S2).

No que diz respeito às competências técnicas, estas implicavam: "conhecimento técnico sobre como intervir (modelos de Intervenção) junto destas crianças" (S7); "desenvolver atividades por forma a explorar o impacto da violência conjugal em menores; compreender o papel do lúdico na intervenção” (S8); revelar "capacidade de observação dos sinais indicadores de existência de disfuncionalidade ou risco/ negligência parental” (S9).

As competências relacionais apontadas pelos técnicos (S1, S4, S5, S8, S9, S10, S11) para o trabalho com as crianças prendiam-se com características pessoais. O técnico devia ser capaz de "escutar, ter flexibilidade, ter abertura à mudança e ter empatia" (S10) e "proporcionar o desenvolvimento de um sentimento de confiança” (S11). Foram ainda referidos outros aspetos como:

"Ser empático, dinâmico e disponível. Tecnicamente saber ouvir, estar atento a pequenos sinais de alerta (dar-lhes a devida importancia), saber interpretar a linguagem não-verbal, informar de forma ajustada conforme a idade e transmitir segurança." (S1).

\subsection{Intervenção desenvolvida com as crianças na casa de abrigo.}

Os técnicos (S1, S2, S3, S5, S6, S8, S9) consideraram "fundamental que a equipa trabalhe com vista a minimizar o impacto que a violência teve sobre a criança e que a ajude a desenvolver a autoestima e autoconfiança" (S5). Os profissionais referiram problemas emocionais e de comportamento, como as áreas mais afetadas nestas crianças aquando da sua entrada na instituição de acolhimento. Era necessário maior cuidado quando se trata da intervenção com crianças que muito frequentemente "precisam de estabilizar emocionalmente" (S1). As crianças que conviveram com a violência sofrem graves alterações nas suas vidas, que podem levar a que se sentissem confusas, sem perceber o porquê da saída de casa e o porquê de abandonar o pai, que na maioria das vezes era o agressor (S1, S3, S5, S8).

Segundo os técnicos (S2, S3, S6, S9), as crianças manifestavam necessidade de serem apoiadas sobretudo a nível psicológico "de forma a facilitar compreender e ultrapassar as situações vivenciadas, lidando com as mesmas de uma forma mais ajustada" (S6). A maioria dos participantes refere que a intervenção individual e a intervenção em grupo eram apropriadas para estas crianças que estiveram expostas à violência interparental. 
Considero que é extremamente importante que haja intervenção individual (apoio elou acompanhamento psicológico) e em grupo. Para dar oportunidade da criança ter o seu espaço (individual) para adquirir confiança e poder expor as suas angústias e medos, bem como é importante o espaço grupal de partilha e de modelagem. (S2)

A experiência numa das casas de abrigo de intervenção com as crianças foi retratada como muito positiva - "costuma funcionar muito bem, a intervenção em grupo, eles costumam aderir bastante bem e tem funcionado" (S3). No entanto, existem casas de abrigo, nas quais não era realizada intervenção com as crianças, visto que os técnicos já faziam acompanhamento às mães, pelo que consideram que não seria ético que o profissional acompanhasse mães e filhos. Assim, o que algumas casas de abrigo optaram, foi pelo encaminhamento das crianças para outros serviços.

Encaminhando as crianças para a consulta de psicologia do centro de saúde elou hospital; já que a psicóloga da casa de abrigo atende as mães destas crianças, $e$ as utentes do centro de atendimentos, desta forma não seria ético estar a intervir diretamente na criança. (S9)

A intervenção direta com as crianças era menor, sendo realizada de acordo com as necessidades evidenciadas pelas mesmas. Assim foram "desenvolvidas diferentes atividades meramente lúdicas para ajudar a normalizar os efeitos negativos do contexto de violência que vivenciaram" (S1). Outros exemplos do apoio prestado foram:

a equipa técnica procede a uma avaliação informal das necessidades da criança, fazendo os encaminhamentos e acompanhamentos necessários no que respeita a serviços de saúde, estabelecimentos educativos, aquisição de vestuário, acompanhamento psicológico, intervenções da Comissão de Proteção de Crianças e Jovens, entre outros (S4).

Este trabalho era muito importante, visto que só após a realização do levantamento das necessidades das crianças, é que estes realizavam os encaminhamentos necessários, deixando evidente que muita da intervenção com estas crianças passava por apoio de serviços externos à casa de abrigo. Por exemplo, "Quando se verifica a necessidade de um acompanhamento em consulta de pedopsiquiatria ou psicologia, a criança é encaminhada para serviço específico do serviço nacional de saúde" (S5). 


\subsection{Dificuldades/desafios na intervenção com crianças}

Os técnicos (S1, S4, S5, S8, S9) consideraram as 'dificuldades' uma presença constante no dia-a-dia das casas de abrigo, designadamente por questões que não podiam ser controladas por eles, tais como: a forma como as utentes vivenciavam a experiência do acolhimento, assuntos relacionados com as burocracias institucionais, as consequências provocadas pela exposição à violência e os próprios órgãos judiciais.

As maiores dificuldades prendem-se com a falta de estruturas especificas de apoio/formação aos pais no que concerne às competências parentais; os maus-tratos e negligência de que muitas vezes estas crianças são vítimas por parte das próprias utentes, o que coloca a equipa técnica numa posição difícil de conciliação de intervenções; a falta de celeridade do sistema judicial no que concerne, por exemplo, aos processos de responsabilidades parentais; questões jurídicas relativas à proteção e segurança das crianças face ao agressor da mãe, que muitas vezes é também agressor dos menores; a ineficácia dos sistemas de proteção de crianças e jovens (S4).

A entrada de adultos e crianças na casa de abrigo dava-se numa fase muito crítica e "quanto maior o tempo de exposição à violência, mais difícil se torna o trabalho com as crianças pois é mais difícil atingir os objetivos." (S5). Os técni$\cos (\mathrm{S} 4, \mathrm{~S} 5, \mathrm{~S} 6, \mathrm{~S} 9)$ consideraram que o seu trabalho era dificultado pelo facto de estas mulheres, bem como os seus filhos, se encontrarem bastante afetados a nível emocional ("As saudades que sentem dos familiares, perceberem porque têm que estar acolhidas. Perceberem a dualidade de sentimentos que têm em relação ao progenitor", S2) e pelos comportamentos desajustados provocados pela presença de violência na vida das crianças. Como referiram alguns técnicos:

A maior dificuldade assenta na alteração de comportamentos desajustados, face aos diferentes contextos e promoção de hábitos saudáveis (...) A dificuldade é maior devido à resistência de muitas mães à mudança. (S6);

Em termos de aceitação e integração no contexto casa abrigo, pois implica mudanças e transformações na vida das crianças, e conquistar a confiança destas crianças, que vêm fragilizadas do seu contexto familiar, pelas dinâmicas associadas (S9).

Apenas uma resposta estava relacionada com desafios na intervenção a realizar com estas crianças que passaram por experiências traumatizantes. No caso em concreto a participante propunha a abertura da casa de abrigo a outras práticas, por exemplo, “implementar um programa de intervenção em grupo, especificamente para este tipo de crianças” (S3). 


\section{Sugestões na intervenção com crianças}

Os técnicos (S1, S2, S3, S4, S6, S7, S9, S11) reforçaram a importância da intervenção com crianças acolhidas em casas de abrigo e estão cientes das necessidades institucionais revelando que "apesar dos técnicos poderem intervir com crianças, a intervenção tem que ser sobretudo ao nível das mães pois são estas que vão "educar" e cuidar dos filhos, incutindo neles os valores e formas de estar na vida que praticam e, eventualmente, consideram mais corretos.” (S6)

Os técnicos fizeram sugestões de práticas que lhes parecem apropriadas para ultrapassar os constrangimentos já apontados, como por exemplo, "Seria importante criar protocolos de intervenção (no que concerne a área de psicologia) transversais a todas as casas de abrigo, bem como formação especializada para a equipa técnica, de forma a adequar a intervenção transversalmente, com base científica atualizada" (S2). Algumas das sugestões incluem alterações internas na casa de abrigo - "O ideal era ter um técnico para as utentes e um técnico para as crianças. Ou então algum estagiário com supervisão da minha parte, também seria uma resposta” (S3).

\section{Discussão}

Mesmo em contexto de acolhimento temporário e mais ainda, pela transitoriedade dessa permanência (Bowyer, Swanston e Vetere, 2015), para a receção e a continuidade da estadia numa casa de abrigo para vítimas de violência, esta deve ter pensadas estratégias de atuação. Entre outras, serão usadas certamente estratégias reativas, dada a situação de crise em que se acolhem os seus utentes, cujas necessidades ditam de imediato que a intervenção psicossocial esteja, desde logo, disponível. Os técnicos das casas de abrigo que participaram neste estudo referiram uma prática de intervenção multidisciplinar, que resulta do facto das necessidades às quais se procura dar resposta serem tão diversas (e.g., saúde, emprego, educação dos filhos) e produzirem impacto tanto nas utentes adultas como nas crianças. Constatou-se que a intervenção, embora mais dirigida diretamente às mulheres, contempla algumas propostas que têm como fundamento o sucesso do agregado (Ahsan, 2004; Graham-Bermann, 2001; Magalhães, Morais, \& Castro, 2011), o que inclui necessariamente os filhos. Exemplos disso são as atividades de apoio à parentalidade positiva (Sani, 2008, no prelo) que certas propostas interventivas (e.g., Center for Child and Family Health, 2010; Edleson, Nguyen e Kimball, 2011) sugerem que se incluam pela resposta alargada que dão às necessidades das mães e das crianças acolhidas.

Paralelamente, consideram os técnicos que a intervenção com as crianças é fundamental pela necessidade imediata destas estabilizarem emocionalmente, até porque este acolhimento inesperado pode ameaçar, mais ainda, o seu bem-estar (Chanmugam, 2016). Desta forma, vários dos técnicos, embora não possam, por vezes, concretizar algumas propostas de intervenção mais 
individualizadas com a criança, realizam atividades, muitas delas de caráter lúdico, para trabalhar questões que a literatura revela serem importantes, como a segurança (Poole, Beran e Thurston, 2008; Rivett, Howarth e Harold, 2006), o autoconceito, a autoestima (Graham-Bermann, 2001) e desenvolver todo um conjunto de competências que podem ajudá-las a ter um melhor ajustamento global (Del Valle \& Zurita, 2005; Sani e Caridade, 2016). A função dos técnicos passa necessariamente pela proteção destas crianças, promoção dos seus direitos, prevenção do impacto negativo da violência doméstica no presente e futuro próximo (Amaral e Martins, 2014; Schechter e Knitzer, 2004).

Os técnicos têm a noção clara de que a intervenção encetada, embora de caráter terciário, pode ter implicações muito importantes, no evitar que estas crianças cruzem com o sistema de proteção ou de justiça (Schechter e Knitzer, 2004) por outras situações de perigo. A experiência adquirida no contacto com crianças em casas de abrigo sensibilizou-os para as necessidades que urge colmatar e dos conhecimentos teóricos e competências técnicas e relacionais que importa possuir para intervir com elas. Por essa razão, importa promover e apoiar a iniciativa dos profissionais na melhoria da sua formação (Sani, no prelo), bem como as tentativas que alguns revelam fazer de implementação de programas e atividades dentro das instituições, como intervenção em grupo (Sani, 2018; Thompson e Trice-Black, 2012). Todavia este investimento pode não ser suficiente para colmatar as dificuldades apuradas, pois como reconhecido em alguns estudos (Magalhães, Morais, \& Castro, 2011) há geralmente uma contradição inevitável, pois a preparação dos técnicos e o incremento de mecanismos de empowerment (empoderamento) das mães, não se traduz necessariamente numa capacitação e autonomia destas, no caso para responder às necessidades dos filhos.

Os técnicos referem igualmente a conveniência de uma intervenção individual com as crianças acolhidas em casa de abrigo. Há situações em que, carecendo de um apoio mais individualizado e na impossibilidade de este ser dado na casa de abrigo, até por uma questão ética e de não sobreposição de funções, é feito um encaminhamento para serviços externos. Os técnicos admitem que a intervenção com crianças em contextos de casa de abrigo é constrangida pela limitação do número de profissionais, que afetos a certas funções não podem responder a tudo, como seria desejado. (Magalhães, Morais, \& Castro, 2011). São necessários mais profissionais e mais elementos que não pertencentes à equipa técnica contribuam para uma eficiente e eficaz resposta social junto das vítimas, evitando-se o risco de uma horizontalidade de funções, que se traduz, por vezes, em alguns destes contextos na assunção de tarefas técnicas por membros não técnicos e vice-versa (Magalhães, Morais, \& Castro, 2011).

Os participantes técnicos neste estudo, convictos da importância de que a intervenção encetada nas casas de abrigo deverá estender-se igualmente às crianças nestas acolhidas, fazem sugestões que permitam alargar essa 
resposta no acolhimento temporário. Neste sentido, alguns técnicos propõem parcerias com serviços e entidades que especificamente podem auxiliar na intervenção especializada com as crianças, enquanto outros mostram-se abertos e procuram implementar algumas atividades para responder às necessidades evidenciadas pelos filhos das utentes da casa. A intervenção em grupo tem-se mostrado eficaz (Sani, 2018), ao mesmo tempo que se trabalham outros aspetos que visam dar suporte às mães e, no geral, favorecer a convivência e bem-estar de todos (Graham-Bermann, 2001).

\section{Conclusão}

As críticas em torno da institucionalização e as consequências produzidas pela mesma (e.g. cortes afetivos, a perda de vínculos, da liberdade, da autonomia, a afetação do desenvolvimento a vários níveis), não se encerram com o debate social e as alterações legislativas trazidas ao acolhimento residencial, mas ajudam-nos a refletir a respeito da qualidade das respostas sociais alternativas para acolhimento à criança exposta direta e/ou indiretamente a situações de violência doméstica. Não obstante as dificuldades de readaptação, o acolhimento temporário de crianças expostas à violência doméstica em casa de abrigo pode traduzir-se numa oportunidade de mudança positiva e desejável ao seu bom desenvolvimento, como pode gerar sentimentos de ameaça e de angústia pelas inúmeras perdas que vivenciam.

Perceber a realidade da intervenção em casas de abrigo na perspetiva dos técnicos que nestas trabalham permite-nos conhecer melhor esta resposta social e compreender como pode a mesma ser melhorada. Em paralelo com os estudos com as vítimas de violência doméstica sobre a sua satisfação com a resposta social casa de abrigo, este estudo permitiu conhecer práticas de intervenção junto desta população, no caso em particular junto das crianças acolhidas, que foram igualmente vítimas e sofreram um impacto negativo no seu ajustamento pela exposição a essa violência doméstica.

Apesar dos esforços e a variedade de serviços oferecidos, a experiência de trabalho neste domínio revela que muitas crianças expostas à violência doméstica têm acesso muito limitado aos mesmos. Fora da resposta de acolhimento residencial apenas algumas crianças são encaminhadas para apoio psicológico, mesmo quando este é disponibilizado. A decisão das vítimas em aceitar recorrer a uma casa de abrigo depende de vários aspetos, entre estes a possibilidade de levar os filhos, sabendo que as características do acolhimento nem sempre permitem que as crianças possam estar. Sendo os filhos um dos motivos que mais influenciam a tomada de decisões das vítimas adultas e sendo evidente a necessidade de a intervenção se estender também às crianças, reforçamos com este trabalho a importância de continuarem a serem feitos esforços para a garantia da proteção e promoção do bem-estar das crianças e suas famílias. 


\section{Referências bibliográficas}

AHSAN, Nilofer (2004), "Domestic Violence and family support programs: Creating opportunities to help young children and their families", in Susan Schechter (org.), Early Childhood, Domestic Violence, and Poverty: Helping Young Children and Their Families, Series Paper \#3, Iowa City, School of Social Work, The University of Iowa, Online], disponível em: https:// clas.uiowa.edu/sites/clas.uiowa.edu.socialwork/files/paper_3.pdf [consultado em: 12/11/17].

AMARAL, Inês e MARTINS, Paula Cristina (2014), “Os problemas de comportamento das crianças em Centros de Acolhimento Temporário. Um contributo para a compreensão da realidade portuguesa”, in Manuela Calheiros e Margarida Garrido (org.), Crianças em risco e perigo: Contextos, investigação e intervenção, Vol. IV, Lisboa, Edições Sílabo, 49-75.

BARDIN, Laurence (2006), Análise de conteúdo, Lisboa, Edições 70.

BOURASSA, Chantal, LAVERGNE, Chatal, DAMANT, Dominique, LESSARD, Geneviève e TURCOTTE, Pierre (2006), "Awareness and detection of the co-occurrence of interparental violence and child abuse: Child welfare worker's perspective", Children and Youth Services Review, 28, 1312-1328.

BOWYER, Laura, SWANSTON, Jennifer e VETERE, Arlene (2015), “ 'Eventually you just get used to it': An interpretative phenomenological analysis of 10-16 year-old girls' experiences of the transition into temporary accommodation after exposure to domestic violence perpetrated by men against their mothers", Clinical Child Psychology and Psychiatry, 20(2), 304-323.

CAMPANÓN, Cristina Bravo (2008), “Menores víctimas de violencia de género: experiencia de intervención en un centro de acogida para víctimas de violencia de género”, Intervención Psicosocial, 17(3), 337-351.

CENTER FOR CHILD AND FAMILY HEALTH (2010), "The needs of children in domestic violence shelters”, EUA, Center for Child and Family Health, [Online], disponível em: https:// childandfamilypolicy.duke.edu/pdfs/projects/CCFH_Toolkit.pdf [consultado em: 12/11/17].

CHANMUGAM, Amy (2016), "Children and young people in domestic violence shelters", Geographies of Children and Young People, 12, 19-43.

CHANMUGAM, Amy (2017), "Children and young people in domestic violence shelters", in Claire Freeman, Paul Tranter e Tracey Skelton (org.), Risk, Protection, Provision and Policy, Geographies of Children and Young People, 12, Singapura, Springer Singapore, 19-43.

CHANMUGAM, Amy e HALL, Kimberly (2012), "Safety planning with children and adolescents in domestic violence shelters", Violence and Victims, 27(6), 831-848.

CORREIA, Ana e SANI, Ana (2015), “As casas de abrigo em Portugal: caraterização estrutural e funcional destas respostas sociais”, Análise Psicológica, 1(XXXIII), 89-96.

CUNNINGHAM, Alison e BAKER, Linda (2004). "What about me: seeking to understand the child's view of violence in the family", London, On: Centre for Children e Families in the Justice Systems of the London Family Court Clinic, [Online], disponível em: http://www.lfcc. on.ca/what_about_me.pdf [consultado em: 12/11/17].

EVANS, Sarah, DAVIES, Corrie e DILILLO, David (2008), "Exposure to domestic violence: A meta-analysis of child and adolescent outcomes”, Aggression and Violent Behavior, 13, 131-140.

DECRETO-LEI n. ${ }^{\circ}$ 323/2000, Regulamenta a Lei 107-1999, Decreto regulamentar n. 1/2006, de 25 de janeiro. (2006), Organização, funcionamento das casas de abrigo, Diário da República, $1^{a}$ série, n. 18, 594-601.

DECRETO REGULAMENTAR n. ${ }^{\circ}$ 2/2018, regula as condições de organização e funcionamento das estruturas de atendi mento, das respostas de acolhimento de emergência e das casas de abrigo que integram a rede nacional de apoio às vítimas de violência doméstica, Diário da República, $1 .^{a}$ série, n. ${ }^{\circ} 17$ de 24 de janeiro de 2018. 
DEL VALLE, Jorge Fernández e ZURITA, Jesús Fuertes (2005), "El acogimiento residencial en la protección a la infancia”, Madrid, Ediciones Pirámide.

DELGADO, Paulo e GERSÃO, Eliana (2018), "O acolhimento de crianças e jovens no novo quadro legal. Novos discursos, novas práticas?” Análise Social, 226, LIII (1. $\left.{ }^{\circ}\right), 112-134$, [Online], disponível em: http://analisesocial.ics.ul.pt/documentos/n226a05.pdf [consultado em: 25/05/18].

EDLESON, Jeffrey, Nguyen, H.T. e Kimball, E. (2011), Honor our voices: A guide for practice when responding to children exposed to domestic violence, Minneapolis, MN, Minnesota Center against Violence and Abuse (MINCAVA), [Online], disponível em: https://calio.dspacedirect.org/handle/11212/1748 [consultado em: 12/11/17].

ERICKSEN, Janet e HENDERSON, Angela (1998), "Diverging realities. Abused women and their children" in Jacquelyn Campbell (Org.), Empowering survivors of abuse. Health care for battered women and their children, Thousand Oaks, Sage Publications, 138-155

FANTUZZO, John e MOHR, Wanda (1999), "Prevalence and effects of child exposure to domestic violence", The Future of Children, Princeton, 9, 21-32.

FARO, Patrícia e SANI, Ana (2014), "Representações de violência doméstica por mulheres vítimas e as respostas pessoais e sociais ao problema", Interconexões, 2(1), 47-64.

FARO, Patrícia e SANI, Ana (2014), "Reconhecimento social da violência doméstica como um problema a combater" in Ana Sani e Laura Nunes (org.), Crime, Justiça e Sociedade. Desafios emergentes e propostas multidisciplinares, Porto, Edições CRIAP, 35-49.

GALIANO, Maria José Justicia e DUARTE, José Cantón (2011), “Conflictos entre padres y conducta agresiva y delictiva en los hijos", Psicothema,23(1), 20-25.

GRAHAM-BERMANN, Sandra (2001), "Designing intervention evaluations for children exposed to domestic violence: Applications of research and theory" in Sandra Graham-Bermann e Jeffrey Edleson (org.), Domestic violence in the lives of children: The future of research, intervention, and social policy, Washington, DC, American Psychological Association, 237267. https://doi.org/10.1037/10408-012

GRAHAM-BERMANN, Sandra e HUGHES, Honore (2003), "Intervention for children exposed to interparental violence (IPV): assessment of needs and research priorities", Clinical Child and Family Psychology Review, 6(3), 189-204.

HOGAN, Fergus e O'REILlY, Máire (2007), "Listening to Children: Children's Stories of Domestic Violence", Department of Health and Children, Office of the Minister for Children [Online], disponível em: http://www.dcya.gov.ie/documents/publications/Listening_childrens_stories_domestic_violence_11_Oct_07.pdf [consultado em: 12/11/17].

HOLT, Stephanie, BUCKLEY, Helen e WHELAN, Sadhbh (2008), "The impact of exposure to domestic violence on children and young people: A review of the literature", Child Abuse \& Neglect, 32, 797-810.

JAFFE, Peter, WOLFE, David e WILSON, Susan (1990). Children of battered woman, USA, Sage Publications.

JOURILES, Ernest, MCDONALD, Renee, SLEP, Amy, HEYMAN, Richard e GARRIDO, Edward (2008), "Child abuse in the context of domestic violence: Prevalence, explanations, and practice implications", Violence and Victims, 23, 221-235.

JOURILES, Ernest, ROSENFIELD, David, MCDONALD, Renee e MUELLER, Victoria (2014), "Child involvement in interparental conflict and child adjustment problems: A longitudinal study of violent families", Journal of Abnormal Child Psychology, 42, 693-704.

INSTITUTO DE SEGURANÇA SOCIAL (2017), CASA 2016 - Relatório de Caracterização Anual da Situação de Acolhimento das Crianças e Jovens, Departamento de Desenvolvimento Social e Programas/ Unidade de Infância e Juventude, Lisboa, Instituto da Segurança Social, I.P. disponível em: http://www.seg-social.pt/documents/10152/15292962/Relatorio_ CASA_2016/b0df4047-13b1-46d7-a9a7-f41b93f3eae7 [consultado em: 25/15/18]. 
Lei n. ${ }^{\circ}$ 107/99, de 3 de agosto, Criação da rede pública de casas de apoio a mulheres vítimas de violência, Diário da República, $1^{\text {a }}$ serie A, nº179 - 3 de agosto de 1999.

Lei n. ${ }^{\circ}$ 112/2009, Estabelece o regime jurídico aplicável à prevenção da violência doméstica, à proteção e à assistência das suas vitimas e revoga a Lei $n .^{\circ} 107 / 99$, de 3 de agosto, e o Decreto -Lein. ${ }^{\circ} 323 / 2000$, de 19 de Dezembro, Diário da República, $1^{\text {a }}$ serie A, nº189 - 16 de setembro de 2009 .

Lei n. ${ }^{\circ}$ 129/2015, Terceira alteração à Lei n. ${ }^{\circ}$ 112/2009, de 16 de setembro, que estabelece o regime jurídico aplicável à prevenção da violência doméstica, à proteção e à assistência das suas vítimas, Diário da República, $1^{\mathrm{a}}$ serie A, nº 172 - 3 de setembro de 2015.

MANDAL, Mahua e HINDIN, Michelle (2015), "Keeping it in the family: Intergenerational transmission of violence in Cebu, Philippines", Maternal Child Health Journal, 19, 598-605.

MARGOLIN, Gayla e VICKERMAN, Katrina (2007), "Posttraumatic stress in children and adolescents exposed to family violence: I. Overview and issues", Professional Psychology: Research and Practice, 38, 613-619.

MARTINS, Paula Cristina (2016), “Acolhimento residencial” in Rui Maia, Laura Nunes, Sónia Caridade, Ana Sani, Rui Estrada, Cristiano Nogueira, Helder Fernandes e Lígia Afonso (Org.), Dicionário Crime, Justiça e Sociedade, Lisboa, Edições Sílabo, 19-20.

MCDONALD, Renee e GRYCH, John (2006), “Young children's appraisals of interparental conflict: Measurement and links with adjustment problems", Journal of Family Psychology, 20(1), 88-99.

MORETTI, Marlene, BARTOlO, Tania, CRAIG, Stephanie, SLANEY, Kate e ODGERS, Candice (2014). "Gender and the transmission of risk: A prospective study of adolescent girls exposed to maternal versus paternal interparental violence", Journal of Research on Adolescence, 24(1), 80-92.

MAGAlHÃES, Maria José, MORAIS, Carminda e Castro, Yolanda Rodríguez (2011), "Organização e funcionamento duma casa de abrigo de solidariedade social", Psicologia $e$ Sociedade, 23, 598-607.

MUÑOZ, Cecilia, BELlO, Maria, SANDOVAL, Sandra, ROMERO, Lucy e NIETO, José (2016), "Relación entre problemas de conducta en adolescentes y conflicto interparental en familias intactas y monoparentales”, Revista Colombiana de Psicología, 25(1), 107-122.

ØVERLIEN, Carolina (2011), "Women's refuges as intervention arenas for children who experience domestic violence", Child Care in Practice, 17, 375-391.https://doi.org/10.1080/1357 5279.2011 .596816

PELED, Einat e Davis, Diane (1995), Groupwork with children of battered woman: A practioner's guide, London, Sage Publications.

POOLE, Ann, BERAN, Tanya e THURSTON, Wilfreda (2008), "Direct and indirect services for children in domestic violence shelters", Journal of Family Violence, 23, 679-686.

RIVETT, Mark, HOWARTH, Emma e HAROLD, Gordon (2009). "'Watching from the stairs': towards an evidence-based practice in work with child witnesses of domestic violence", Clinical Child Psychology and Psychiatry, 1, 103-125.

SAATHFF, Amy e STOFFEL, Elizabeth (1999), "Community-based domestic violence services. Domestic violence and children: Analysis and recommendations". The David and Lucile Packard Foundation. The Future of Children, 9(3), 4-20.

SANI, Ana (no prelo), "Exposição da Criança à Violência Doméstica: (re)conhecimento e (re) ação atuais” in Isabel Dias (org.), Manual de Violência Doméstica, Lisboa, Pactor.

SANI, Ana (2004), “O discurso de crianças expostas à violência interparental - Estudo Qualitativo", Psychologica, 36, 109-130.

SANI, Ana (2008), "Mulher e mãe no contexto de violência doméstica: a experiência de parentalidade", Ex-aequo - Revista da Associação Portuguesa de Estudos sobre as mulheres, 18, $123-133$. 
SANI, Ana (2018). "Intervenção terapêutica em grupo com crianças expostas à violência doméstica" in Ana Sani e Sónia Caridade (org.), Violência, agressão e vitimação: Práticas para a Intervenção, $2^{\mathrm{a}}$ edição, Coimbra, Edições Almedina, 35-57.

SANI, Ana e CARIDADE, Sónia (2016), "Intervenção em contexto de acolhimento institucional com crianças e jovens vítimas de violência" in Ana Sani e Sónia Caridade (org.), Práticas de intervenção na violência e no crime, Lisboa, Pactor, 19-30.

SCHECHTER, Susan e KNITZER, Jane (2004), "Series Introduction” in S. Schechter (org.), Early Childhood, Domestic Violence, and Poverty: Helping Young Children and Their Families, Iowa City, School of Social Work, The University of Iowa, i-v.

SOARES, Liliana e SANI, Ana (2015). "O impacto da exposição à violência interparental nas crianças: variáveis mediadoras”, Revista Psicologia da Criança e do Adolescente, 6(2), 155-169.

STRAUSS, Anselm e CORBIN, Juliet (1998), Basics of qualitative research - Techniques and procedures for developing Grounded Theory, 2nd edition, London, Sage Publications.

THOMPSON, Heather e TRICE-BLACK, Shannon. (2012), "School-based group interventions for children exposed to domestic violence, Journal of Family Violence, 27, 233-241.

WAGAR, Janet e RODWAY, Margaret (1995). "An evaluation of a group treatment approach for children who have witnessed wife abuse", Journal of Family Violence, 10(3), 295-306.

WILliAMSON, Emma (2006), 2005 Survey of Domestic Violence Services Findings. England: Women's Aid Federation of England. 